\title{
Isolamento social: consequências físicas e mentais da inatividade física em crianças e adolescentes
}

\section{Social isolation: physical and mental consequences of physical inactivity in children} and adolescents

AUTORES
Públio Gomes Florêncio Júnior ${ }^{1,2}$ (D)
Rone Paiano ${ }^{3,4}$ (D)
André dos Santos Costa ${ }^{1}$ (D)
1 Universidade Federal de Pernambuco, Centro de
Ciências da Saude, Departamento de Educação
Física, Recife, Pernambuco, Brasil.
2 Centro Universitário Mauricio de Nassau, Centro
de Saúde, Departamento de Educação Física,
Recife, Pernambuco, Brasil.
3 Universidade Presbiteriana Mackenzie, Centro de
Ciências Biológicas e da Saúde, Departamento de
Educação Física, São Paulo, São Paulo, Brasil.
4 Faculdade das Américas, Departamento de
Educação Física, São Paulo, São Paulo, Brasil.
CONTATO

Públio Gomes Florêncio Júnior.

publio_@hotmail.com

Rua Osvaldo Guimaraes, 45, apto 103, Iputinga, Recife, Pernambuco, Brasil.

CEP: 50670-330.

DOI

$10.12820 /$ rbafs. $25 \mathrm{e} 0115$

\section{(c) BY}

Este trabalho está licenciado com uma Licença Creative Commons - Atribuição 4.0 Internacional.

\begin{abstract}
RESUMO
Este documento de opinião apresenta as possíveis consequências para saúde física e mental de crianças e adolescentes, que ficarão longos períodos em quarenta por conta da pandemia do COVID-19. A quarentena é uma estratégia necessária e implementada por vários governos em diversos países, no entanto, o isolamento social pode diminuir de forma profunda os níveis de atividade física e aumentar os níveis de comportamento sedentário em jovens que estão sem aulas presenciais neste momento. Tudo isso aumenta o risco de ganho de peso, obesidade, ansiedade, depressão e até modificações na estrutura cerebral dessa população. Acreditamos que este documento indica possibilidades de aumentar os níveis de atividade física em crianças e adolescentes neste período de isolamento social.

Palavras-chave: Criança; Comportamento sedentário; Saúde mental; Quarentena.

\section{ABSTRACT}

This opinion document presents as possible consequences for the physical and mental health of children and adolescents, who are in long periods of quarantine due to the COVID-19 pandemic. Quarantine social isolation a strategy applied and implemented by several countries, however, social isolation can profoundly decrease levels of physical activity and increase levels of sedentary behavior in young people who are currently out of school. All of this increases the risk of weight gain, obesity, anxiety, depression and even changes in the brain structure of this population. We believe that this document indicates possibilities for increasing levels of physical activity in children and adolescents in this period of social isolation.
\end{abstract}

Keywords: Child; Sedentary behavior; Mental health; Quarantine.
Atendendo à chamada de artigos para a edição temática "Exercício Físico/Atividade Física e o COVID-19", apresentamos esta carta que discute as possíveis consequências para saúde física e mental de crianças e adolescentes, que ficarão longos períodos em quarentena inativos por consequência da pandemia do COVID-19.

Com o surgimento da pandemia do COVID-19, a Organização Mundial da Saúde (OMS) recomendou o isolamento social como estratégia mais eficiente para evitar contaminação e mortes, visto não haver tratamento comprovadamente eficaz até o momento. Nesse sentido, vários países adotaram trabalhos em formato home-office, fechamento de fábricas, comércio, escritórios, clubes e espaços de pratica de exercício físico, assim como a suspensão das atividades de ensino.

Apesar de ser uma medida necessária, espera-se que isolamento social gere efeitos psicológicos negativos, podendo se estender para consequências físicas e mentais em diferentes faixas etárias e, em especial, nas crianças e adolescentes que deixam de frequentar a escola. De fato, é provável que jovens permaneçam mais tempo sentados em atividades sedentárias em jogos online, assistindo TV e até em aulas remotas, o que, consequentemente, acarretará uma redução dos níveis de atividade física ${ }^{1}$. Nesse sentido, parece que a inserção de momentos ativos em casa são necessários, pois quanto menos tempo sedentário, melhor será a qualidade vida relacionada a saúde em crianças e ado- 
lescentes. Outrossim, a atividade física tem um papel mediador na associação entre sintomas depressivos e comportamento sedentário baseado em tela, assim como, quando praticada de forma vigorosa parece minimizar as relações entre ansiedade e comportamento sedentário baseado em tela em crianças e adolescentes ${ }^{2}$.

O comportamento sedentário também pode afetar negativamente as estruturas cerebrais em crianças com sobrepeso/obesidade. Em recente estudo foi observado que maiores tempos destinados a assistir TV associou-se com menor volume de massa cinzenta em seis regiões do cérebro, mais tempo jogando videogame foi associado a redução de massa cinzenta em três regiões cerebrais e o tempo total de comportamento sedentário associou-se à redução de massa cinzenta em duas regiões do cérebro. Tais achados sugerem que crianças com sobrepeso/obesidade podem ter suas funções cognitivas prejudicadas pelo comportamento sedentário, devido a possíveis alterações na estrutura cerebral ${ }^{3}$. Esses resultados aumentam ainda mais a preocupação com a saúde durante o isolamento social, uma vez que baixos níveis de atividade física e elevado tempo de tela estão associados a riscos aumentados para o sobrepeso e obesidade. Portanto, acreditamos que esses elementos dão suporte à necessidade de aumentar os níveis de atividade física em crianças e adolescentes neste período de isolamento social.

A prática de atividade física regular é importante para manutenção do peso adequado em estudantes, além de proporcionar benefícios para a cognição. Interessantemente, uma única sessão de exercícios físicos pode gerar efeitos positivos sobre funções cognitivas importantes, assim como, quando realizados cronicamente, pode promover adaptações em estruturas cerebrais e na plasticidade sináptica, importantes para a cognição ${ }^{4}$.

Uma possibilidade encontra-se no uso de exergames, jogos virtuais com maior demanda física que os videogames tradicionais, pois podem melhorar a saúde através do aumento dos níveis de atividade física. Além das demandas físicas, crianças e adolescentes com sobrepeso e obesidade podem melhorar os aspectos psicológicos, especificamente a autoestima e a autoeficácia, após a pratica de exergames ${ }^{5}$. Outras alternativas para manter-se ativo durante o isolamento social merecem destaque como exercitar-se através de videoaulas, realizar tarefas domésticas com exigência física, manter-se mais tempo em pé e fazer pausas curtas durante o dia para caminhar dentro de casa, além da prática de relaxamento.

Neste contexto alguns atores serão importantes para o maior engajamento de crianças e adolescentes. Em primeiro lugar, os familiares são de vital importância para o maior engajamento na prática de exercício físico de crianças e adolescentes durante o IS. Em segundo lugar, os professores de educação física que podem ser acessados de forma remota pelos ambientes viabilizados pelas escolas ON-LINE ou mesmo por meio de redes sociais publicando vídeos e sugerindo atividades. E, por último, governantes devem apoiar projetos que estimulem a prática de atividade física para esta população, baseados na literatura científica e considerando os aspectos culturais, sociais e econômicos, sem deixar de respeitar as estratégias de isolamento social devido à pandemia de COVID-19.

\section{Conflito de interesse}

Os autores declaram não haver conflito de interesses.

\section{Contribuição dos autores}

Florêncio Júnior FJ e Costa AS, participaram da concepção inicial do estudo, redação e revisão crítica do texto e aprovação da versão final. Paiano $R$, foi responsável pela busca da literatura e revisão crítica do texto e aprovação da versão final.

\section{Referências}

1. Lourenço CLM, de Souza TF, Mendes EL. Relationship between smartphone use and sedentary behavior: a schoolbased study with adolescents. Rev Bras Ativ Fís Saúde. 2019;24:e078.

2. Zink J, Belcher BR, Imm K, Leventhal AM. The relationship between screen-based sedentary behaviors and symptoms of depression and anxiety in youth: a systematic review of moderating variables. BMC Public Health. 2020;20:472.

3. Zavala-Crichton JP, Esteban-Cornejo I, Solis-Urra P, MoraGonzalez J, Cadenas-Sanchez C, Rodriguez-Ayllon M, et al. Association of sedentary behavior with brain structure and intelligence in children with overweight or obesity: The Active Brains project. J Clin Med. 2020;9(4):1101.

4. Merege Filho CAA, Alves CRR, Sepúlveda CA, Costa AS, Lancha Junior AH, Gualano B. Influência do exercício físico na cognição: Uma atualização sobre mecanismos fisiológicos. Rev Bras Med Esporte. 2014;20(3):237-41.

5. Andrade A, Correia CK, Coimbra DR. The psychological effects of exergames for children and adolescents with obesity: a systematic review and meta-analysis. Cyberpsychology Behav Soc Netw. 2019;22(11):724-35.

Recebido: $12 / 05 / 2020$ Aprovado: 02/06/2020 